\title{
Tissue Factor in the myocardium: Evidence of roles in haemostasis and inflammation
}

\author{
Andrew D. Mumford ${ }^{\mathrm{a}, *}$ and John H. McVey ${ }^{\mathrm{b}}$ \\ ${ }^{a}$ Department of Haematology, Bristol Royal infirmary, Bristol, BS2 8HW, UK \\ ${ }^{\mathrm{b}}$ Haemostasis and Thrombosis, MRC Clinical Sciences Centre, The Faculty of Medicine, Imperial College, \\ Hammersmith Hospital Campus, Du Cane Road, London, W12 ONN, UK
}

\begin{abstract}
The interaction between cell-surface tissue factor (TF) and the plasma coagulation factor VII (FVII) initiates the coagulation network that leads to the generation of thrombin and the formation of a fibrin clot. Thrombin also activates cellular protease activated receptors (PARs) through which it activates components of the inflammatory pathway. TF is expressed constitutively by cardiomyocytes and evidence from mice transgenic for a human TF mini-gene that express very low levels of human TF suggests that the TF-FVII interaction is critical for haemostasis within the heart. Pathological contact between TF and FVII may occur in the heart during ischaemia-reperfusion (I-R) injury and this may lead to activation of coagulation and thrombin generation. Evidence from animal models now suggests that thrombin is an important mediator of inflammation in I-R injury. The coagulation pathway therefore represents a novel therapeutic target for intervention in the prevention of I-R injury.
\end{abstract}

Keywords: Tissue factor, blood coagulation, inflammation, cardiomyocytes, ischaemia-reperfusion injury

\section{Introduction}

The transmembrane glycoprotein tissue factor (TF) is the cellular receptor and co-factor for the plasma coagulation factor VII (FVII) and the activated form of FVII, termed FVIIa. Binding of FVII to membrane bound TF leads to activation of FVII to FVIIa and the resulting TF/FVIIa complex activates coagulation factors IX and X (FIX and FX). In the absence of its cofactor, activated factor $\mathrm{V}$ (FVa), FXa generates only trace amounts of thrombin from prothrombin. The thrombin formed in this initiation stage of coagulation is insufficient to cause significant fibrin polymerisation but instead, back-activates the co-factors FV and factor VIII (FVIII). In this amplification stage of coagulation, FVIIIa forms a complex with FIXa and activates increasing quantities of FX. In turn, FXa forms a complex with FVa, and generates sufficiently large quantities of

*Corresponding author: Dr. AD Mumford, Department of Haematology, Bristol Royal infirmary, Bristol, BS2 8HW, UK. Tel.: +44 117928 2655; Fax: +44 117928 4036; E-mail: a.mumford@ bristol.ac.uk. thrombin to allow fibrin clot formation (Fig. 1). Although thrombin is the protease directly responsible for the generation of a fibrin clot, the interaction between TF and FVII is the initiating event in the coagulation pathway and is an important site at which haemostasis is regulated [1].

Inappropriate activation of coagulation is prevented in living organisms by maintaining an anatomical separation between TF on the surface of cells and FVII in the plasma. TF is expressed constitutively at high levels by cells of the vascular adventitia and in tissues that delimit organ and body boundaries [2]. In contrast, cells within the intravascular space normally show absent or very low level TF expression. Disruption of the endothelial lining of blood vessels may result in contact between TF and FVII and so may lead to activation of the coagulation pathway and the generation of thrombin [3]. The importance of avoiding inappropriate contact between TF and FVII is illustrated by pathological events such as disseminated intravascular coagulation in patients with sepsis from gram-negative bacteria. In this situation, coagulation may be activated if TF expression is induced on monocytes by bacte- 


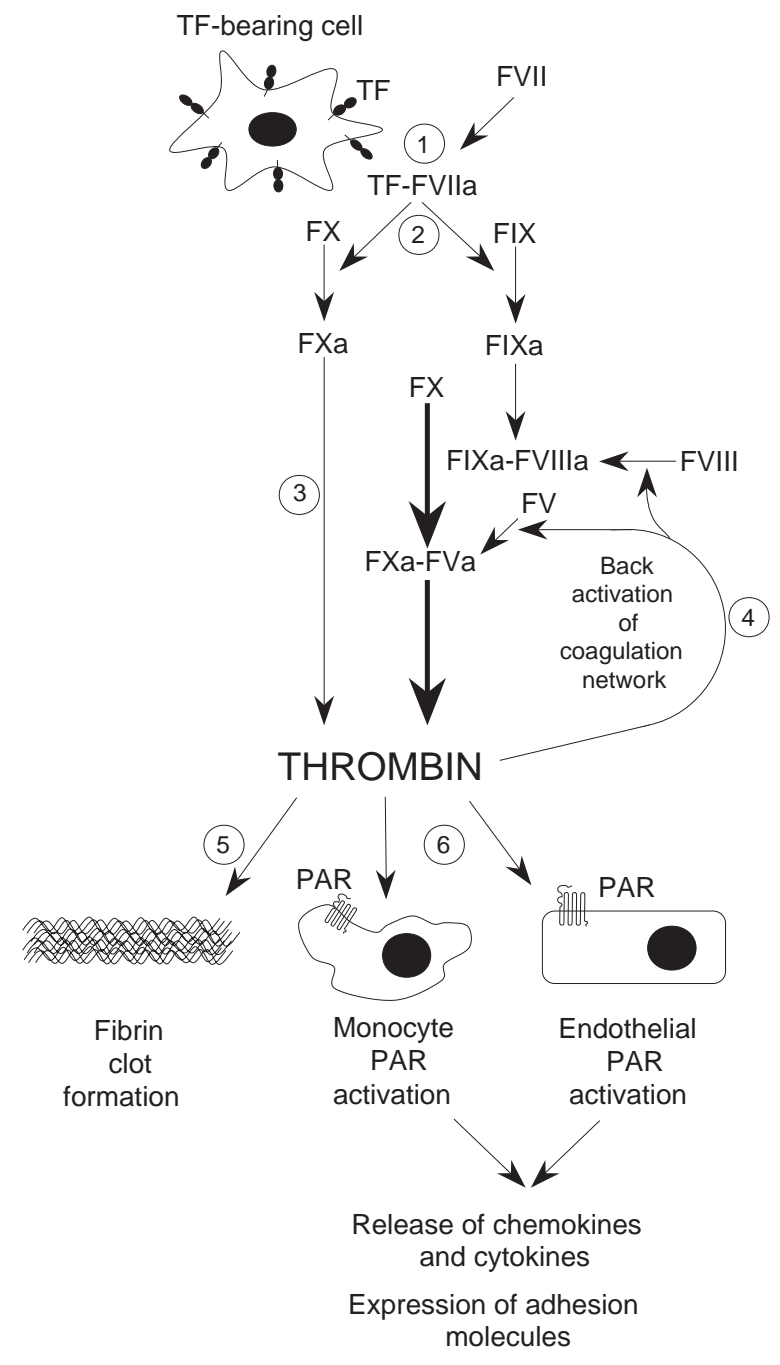

Fig. 1. The coagulation pathway is initiated by exposure of coagulation factor VII (FVII) to cells that express the integral membrane protein tissue factor (TF; 1 ). Activation of FVII to the active protease FVIIa in association with TF on the cell surface activates coagulation factor $\mathrm{X}(\mathrm{FX})$ to form $\mathrm{FXa}(2)$ which in turn generates the active protease thrombin (3). In most tissues, the small quantities of thrombin generated early in the coagulation pathway are insufficient to generate a fibrin clot. Instead, thrombin back-activates factors VIII and V (FVIII and FV) which then serve as cofactors to activated factor IX (FIXa) and FXa respectively, (4). This leads to greatly increased generation of FXa and thrombin. Only then can thrombin cleave fibrinogen to form fibrin (5) and activate inflammatory pathways by activating protease-activated receptors (PARs) on target cells such as monocytes and endothelium (6). Expression of large quantities of TF in myocardium may enable sufficient thrombin generation to enable fibrin formation and activation of inflammatory pathways without requiring back-activation of FV and FVIII.

rial lippopolysaccharide [4]. Similarly, coronary artery thrombosis causing myocardial infarction or unstable angina may follow the exposure of $\mathrm{TF}$ on activated macrophages within atheromatous plaques to FVII in plasma after plaque rupture [5].

In addition to its well-established role as the initiator of coagulation, TF has now also been implicated as a mediator in the inflammatory pathway. Important evidence for this comes from observations that in vitro, pro-inflammatory stimuli up-regulate expression of TF on cells of the vasculature such as endothelium or monocytes [6]. TF is itself an important stimulus of the inflammatory response in disorders such as gramnegative sepsis, connective tissue disease, glomerulonephritis or the systemic inflammatory response to trauma [7-10].

Although, there is some evidence of a direct signalling role for the TF molecule itself [11], many of the pro-inflammatory activities of TF appear to be mediated by a group of cell-surface receptors termed the protease-activated receptors (PARs). PARs are a class of G-protein-coupled receptors that are activated through limited cleavage by a protease so that a new amino terminus is exposed. The new amino-terminus then acts as a tethered ligand that induces signalling through intramolecular binding [12]. Of the 4 known human members of this receptor class, PAR-1, PAR3 and PAR-4 are activated by thrombin [12-14] and PAR-2 by trypsin, FVIIa and FXa [15]. PAR-1 is also activated by activated protein C [16]. Although PAR-1 and PAR-2 stimulate the expression of a wide range of common genes in vascular cells, there is not complete concordance. For example, the mRNA for monocyte chemoattractant protein (MCP-1) is induced by stimulation of PAR-1 but not PAR-2 [16].

The dominant PAR ligand in the human inflammatory response appears to be thrombin and through the PARs, thrombin activates endothelium and monocytes to express adhesion molecules and to release cytokines and chemokines [15,17] (Fig. 1). The coagulation and inflammatory pathways are therefore closely linked and share the common features that they may be initiated by the interaction of TF and FVII and that the effector protease is thrombin. Although both pathways require the generation of thrombin, the pro-inflammatory actions of thrombin do not require the generation of fibrin. Similarly, the pro-coagulant activity of thrombin on fibrin generation does not require the presence of PARs.

\section{What is the pattern of tissue factor expression in the myocardium?}

TF is expressed on cells of the cardiovascular system during the early stages of organogenesis in both 
human and murine development. However, by embryonic day 9.5 in mice and at a comparable developmental stage in human embryos, TF protein and mRNA is demonstrable in the primitive heart [18]. In fully developed hearts, TF is expressed on adventitial cells of the coronary vasculature as in other tissues. However, cardiomyocytes also show high levels of constitutive expression of both antigenic and functional TF [2,4,19]. TF is expressed at greatest levels by contractile cardiomyocytes of the ventricles. Cardiomyocytes from the atria and cells of the cardiac electrical conducting system show lower level expression [20]. At ultrastructural level, TF is distributed on the cardiomyocyte sarcolemma but is concentrated at the intercalated discs between adjacent cardiomyocytes [2,20]. Since the intercalated discs contain the fasciae adherents into which the actin cytoskeleton inserts [21], this raises the possibility that TF may contribute to the structural integrity of the cardiomyocytes [20]. A structural association between TF and actin binding protein has previously been demonstrated in epithelial cells [22,23].

\section{What are the effects of low-level tissue factor expression in the heart?}

In transgenic mice in which the $\mathrm{TF}$ gene has been disrupted, approximately $90 \%$ of the TF-null embryos die by 10.5 days post-coitum from abnormal bleeding or developmental abnormalities in the vasculature [2426]. However, if these mice are further manipulated to express human TF at very low levels by insertion of a TF 'mini-gene', most survive until term [27]. After birth, these 'low-TF' mice show a shortened lifespan in part because of an increased bleeding tendency at sites such as brain, lung and gastrointestinal tract. However, a consistent additional abnormality in these mice is haemosiderin deposition and fibrosis in the myocardium consistent with recurrent intra-cardiac bleeding [28]. The same changes are found in mice genetically modified to express low levels of FVII, but not in those that express low levels of coagulation FIX [29].

FIX and other components of the amplification stage of the coagulation pathway are vital for haemostasis in most tissues since they form an important backactivation loop by which thrombin generation is enhanced (Fig. 1). However, computer-generated and in vitro models of blood coagulation suggest that at very high concentrations of TF, the TF/FVIIa complex can generate enough thrombin for fibrin clot formation without needing amplification through the activation of the co-factors FV and FVIII [30]. This raises the interesting possibility that the high levels of TF in the myocardium are essential for cardiac haemostasis by providing a direct means of generating high levels of thrombin. This mechanism may be disrupted in both low-TF and low-FVII mice and consequently, these strains suffer intra-cardiac bleeds [28]. It is not yet clear whether data from other 'low-coagulation factor' mouse models will support this hypothesis [31].

\section{Does myocardial tissue factor contribute to inflammatory pathways?}

The ability of TF to initiate both coagulation and inflammatory pathways in other tissues raises the possibility that TF expressed in the myocardium may also have a dual role. One circumstance in which inflammatory pathways are activated in the myocardium is following restoration of blood flow after cardiac ischaemia. This common clinical scenario is termed myocardial ischaemia-reperfusion (I-R) injury and its consequences may range clinically from transient dysfunction of myocardial contractility to myocardial infarction [32]. These events may be a source of significant morbidity after interventions such as pharmacological thrombolysis for coronary artery thrombosis, coronary artery bypass surgery or angioplasty [33]. The pathophysiological events that underlie myocardial I-R injury include activation of monocytes, neutrophils and endothelium within the coronary circulation leading to the expression of adhesion molecules and the local release of pro-inflammatory cytokines and chemokines [34-37]. I-R injury may itself cause ischaemia and even infarction of the myocardium through plugging of the coronary microvasculature with aggregates of activated leucocytes [38].

As the pathophysiological events that initiate myocardial I-R injury have become clearer, TF has emerged as a potential mediator of inflammation. For example, oxygen free radicals are generated in the coronary circulation during reperfusion of ischaemic myocardium [39]. Oxygen free radicals also induce TF expression in cultured rabbit coronary endothelial cells [40]. TF pro-coagulant activity has been shown to increase in the coronary circulations of isolated rabbit hearts reperfused after ischaemia and this increase could be abolished by pre-treating the myocardium with oxygen radical scavengers [40]. In an open-chest rabbit model of myocardial I-R injury, reperfusion after only 5 minutes of ischaemia was also associated with 
increased TF activity in the coronary vessels. The I-R injury in this model was not associated with ultrastructural changes in the coronary vasculature [39] so the likely source of this increased TF activity was the coronary endothelium rather than the cardiomyocytes [40]. Importantly, myocardial blood flow in the ischaemic area of the rabbit hearts during reperfusion could be improved by administration of a blocking anti-TF antibody [40]. A similar therapeutic benefit was achieved with human FVII that had been modified so that it retained the ability to bind TF but could not activate downstream components of the coagulation pathway (active site inhibited FVII) [41,42].

In an alternative model of myocardial I-R injury, rabbit hearts reperfused after 45 minutes of ischaemia show regions of both infarcted and non-infarcted tissue within the ischaemic territory [43]. In this circumstance, no increase in endothelial TF expression could be demonstrated in the coronary microvasculature from the non-infarcted ischaemic region. Instead, cardiomyocytes, which already show constitutive expression of TF, showed increased TF mRNA and antigen [44]. In this I-R injury model, there was ultrastructural evidence of disruption of the coronary vascular endothelial barrier in the non-infarcted area and deposition of fibrin in the myocardium [44]. These findings suggest that I-R injury may also be associated with extravasation of clotting factors, activation of coagulation by TF on cardiomyocytes and extravascular generation of fibrin $[44$, 45].

The effects of pharmacologically manipulating different components of the coagulation pathway have also been assessed in rabbit hearts reperfused after 45 minutes of ischaemia. Inhibition of TF with a blocking anti-TF antibody and inhibition of thrombin with hirudin during reperfusion both reduced the extent of myocardial infarction within the ischaemic territory. Inhibition of thrombin with hirudin also reduced neutrophil infiltration and secretion of the chemokines interleukin-8 (IL-8) and MCP-1 [44]. Although depletion of fibrinogen with ancrod abolished fibrin deposition in the myocardium it did not reduce infarct size. These findings suggest that both TF and thrombin have a role in the pathogenesis of myocardial I-R injury. They also suggest that although myocardial fibrin deposition is a feature of I-R injury, the generation of fibrin is not necessary for its pathogenesis.

Thrombin is known to stimulate endothelial cells to secrete the chemoattractants IL- 8 and MCP-1 [46, 47] and to express intercellular adhesion molecule-1 (ICAM-1) and P-selectin [48]. These are necessary for recruitment, adhesion and extravasation of neutrophils in the inflammatory pathway that are an important cause of ischaemia and infarction in I-R injury $[49,50]$. The finding that the secretion of IL- 8 and MCP-1, the extent of neutrophil infiltration and the size of myocardial infarction could all be reduced by thrombin inhibition in the rabbit myocardial I-R injury model is persuasive evidence that thrombin is a key mediator. PAR-1 is expressed on coronary vascular endothelium and smooth muscle cells [47] and this receptor mediates the pro-inflammatory actions of thrombin in other inflammation models [51]. It is tempting to speculate that the PARs are also important in myocardial I-R injury. Preliminary data is now available that suggests that a transgenic mouse strain that does not express PAR-1 appears to be protected against myocardial I-R injury [45]. Further phenotypic characterisation of this strain and studies involving pharmacological inhibition of the PARs in other I-R injury models are required to clarify their role.

\section{Conclusion}

Constitutive expression of TF on cardiomyocytes suggests an important role for TF or TF-dependent pathways in cardiac function. Phenotypic evidence from 'low-TF' transgenic mice suggests that expression of high concentrations of TF appear to be essential for local haemostasis. Possibly because of the unique mechanical demands placed on the coronary microvasculature, the myocardium may require a specialised robust local haemostatic system. TF expression on cardiomyocytes may provide this by acting as a secondary haemostatic barrier outside the coronary vascular adventitia. However, although the high constitutive expression of TF on cardiomyocytes may be necessary for haemostasis, it may also render the myocardium vulnerable to inflammatory stimuli by permitting the rapid local generation of thrombin. The role of the PARs in mediating the cellular responses to thrombin in the myocardium remains unclear and requires further experimental clarification.

Although evidence for a role of TF-dependent thrombin generation in animal models of I-R injury is compelling, evidence of involvement in human disease is more limited [52]. It is already established that the therapeutic use of hirudin reduces cardiovascular death, new myocardial infarction and re-occlusion rates in patients undergoing pharmacological thrombolysis [53, 54]. This raises the exciting possibility that pharma- 
cological manipulation of other parts of the common coagulation-inflammatory pathway may also offer therapeutic benefit.

\section{References}

[1] J.H. McVey, Tissue factor pathway, Baillieres Best Pract Res Clin Haematol 12 (1999), 361-372.

[2] T.A. Drake, J.H. Morrissey and T.S. Edgington, Selective cellular expression of tissue factor in human tissues. Implications for disorders of hemostasis and thrombosis, Am J Pathol 134 (1989), 1087-1097.

[3] T.S. Edgington, N. Mackman, K. Brand and W. Ruf, The structural biology of expression and function of tissue factor, Thromb Haemost 66 (1991), 67-79.

[4] N. Mackman, M.S. Sawdey, M.R. Keeton and D.J. Loskutoff, Murine tissue factor gene expression in vivo. Tissue and cell specificity and regulation by lipopolysaccharide, Am J Pathol 143 (1993), 76-84

[5] V. Toschi, R. Gallo, M. Lettino et al., Tissue factor modulates the thrombogenicity of human atherosclerotic plaques, Circulation 95 (1997), 594-599.

[6] N. Mackman, Regulation of tissue factor gene expression in human monocytic and endothelial cells, Haemostasis 26(1) (1996), 17-19.

[7] A.A. Creasey, A.C. Chang, L. Feigen, T.C. Wun, F.B. Taylor, Jr. and L.B. Hinshaw, Tissue factor pathway inhibitor reduces mortality from Escherichia coli septic shock, J Clin Invest 91 (1993), 2850-2860.

[8] S. Gando, T. Kameue, S. Nanzaki, T. Hayakawa and Y. Nakanishi, Participation of tissue factor and thrombin in posttraumatic systemic inflammatory syndrome, Crit Care Med $\mathbf{2 5}$ (1997), 1820-1826.

[9] F.B. Taylor, Jr. Studies on the inflammatory-coagulant axis in the baboon response to E. coli: regulatory roles of proteins $\mathrm{C}$, S, C4bBP and of inhibitors of tissue factor, Prog Clin Biol Res 388 (1994), 175-194.

[10] P.G. Tipping, J.H. Erlich, J. Apostolopoulos, N. Mackman, D. Loskutoff and S.R. Holdsworth, Glomerular tissue factor expression in crescentic glomerulonephritis. Correlations between antigen, activity, and mRNA, Am J Pathol 147 (1995), 1736-1748.

[11] W. Konigsberg, D. Kirchhofer, M.A. Riederer and Y. Nemerson, The TF: VIIa complex: clinical significance, structurefunction relationships and its role in signaling and metastasis, Thromb Haemost 86 (2001), 757-771.

[12] T.K. Vu, D.T. Hung, V.I. Wheaton and S.R. Coughlin, Molecular cloning of a functional thrombin receptor reveals a novel proteolytic mechanism of receptor activation, Cell 64 (1991), 1057-1068.

[13] H. Ishihara, A.J. Connolly, D. Zeng et al., Protease-activated receptor 3 is a second thrombin receptor in humans, Nature 386 (1997), 502-506.

[14] W.F. Xu, H. Andersen, T.E. Whitmore et al., Cloning and characterization of human protease-activated receptor 4, Proc Natl Acad Sci USA 95 (1998), 6642-6646.

[15] S.R. Coughlin, How the protease thrombin talks to cells, Proc Natl Acad Sci USA 96 (1999), 11023-11027.

[16] M. Riewald, R.J. Petrovan, A. Donner, B.M. Mueller and W. Ruf, Activation of endothelial cell protease activated receptor 1 by the protein C pathway, Science 296 (2002), 1880-1882.
[17] C.T. Esmon, Inflammation and thrombosis, J Thromb Haemost 1 (2003), 1343-1348.

[18] T. Luther, C. Flossel, N. Mackman et al., Tissue factor expression during human and mouse development, Am J Pathol 149 (1996), 101-113.

[19] R.A. Fleck, L.V. Rao, S.I. Rapaport and N. Varki, Localization of human tissue factor antigen by immunostaining with monospecific, polyclonal anti-human tissue factor antibody, Thromb Res 59 (1990), 421-437.

[20] T. Luther, D.D. Dittert, M. Kotzsch et al., Functional implications of tissue factor localization to cell-cell contacts in myocardium, J Pathol 192 (2000), 121-130.

[21] M.S. Forbes and N. Sperelakis, Intercalated discs of mammalian heart: a review of structure and function, Tissue Cell 17 (1985), 605-648.

[22] I. Ott, E.G. Fischer, Y. Miyagi, B.M. Mueller and W. Ruf, A role for tissue factor in cell adhesion and migration mediated by interaction with actin-binding protein 280, J Cell Biol 140 (1998), 1241-1253.

[23] M. Muller, S. Albrecht, F. Golfert et al., Localization of tissue factor in actin-filament-rich membrane areas of epithelial cells, Exp Cell Res 248 (1999), 136-147.

[24] J.R. Toomey, K.E. Kratzer, N.M. Lasky and G.J. Broze, Jr., Effect of tissue factor deficiency on mouse and tumor development, Proc Natl Acad Sci USA 94 (1997), 6922-6926.

[25] P. Carmeliet, L. Moons, M. Dewerchin et al., Insights in vessel development and vascular disorders using targeted inactivation and transfer of vascular endothelial growth factor, the tissue factor receptor, and the plasminogen system, Ann N Y Acad Sci 811 (1997), 191-206.

[26] T.H. Bugge, Q. Xiao, K.W. Kombrinck et al., Fatal embryonic bleeding events in mice lacking tissue factor, the cellassociated initiator of blood coagulation, Proc Natl Acad Sci USA 93 (1996), 6258-6263.

[27] G.C. Parry, J.H. Erlich, P. Carmeliet, T. Luther and N. Mackman, Low levels of tissue factor are compatible with development and hemostasis in mice, J Clin Invest 101 (1998), 560-569.

[28] R. Pawlinski, A. Fernandes, B. Kehrle et al., Tissue factor deficiency causes cardiac fibrosis and left ventricular dysfunction, Proc Natl Acad Sci USA 99 (2002), 15333-15338.

[29] H.F. Lin, N. Maeda, O. Smithies, D.L. Straight and D.W. Stafford, A coagulation factor IX-deficient mouse model for human hemophilia B, Blood 90 (1997), 3962-3966.

[30] S. Butenas, K.E. Brummel, R.F. Branda, S.G. Paradis and K.G. Mann, Mechanism of factor VIIa-dependent coagulation in hemophilia blood, Blood 99 (2002), 923-930.

[31] J.H. McVey, Your bleeding heart: lessons from low tissue factor expression in mice, Trends Pharmacol Sci 24 (2003), 269-272.

[32] K. Matsumura, R.W. Jeremy, J. Schaper and L.C. Becker, Progression of myocardial necrosis during reperfusion of ischemic myocardium, Circulation 97 (1998), 795-804.

[33] S. Verma, P.W. Fedak, R.D. Weisel et al., Fundamentals of reperfusion injury for the clinical cardiologist, Circulation $\mathbf{1 0 5}$ (2002), 2332-2336.

[34] E.D. Verrier and E.N. Morgan, Endothelial response to cardiopulmonary bypass surgery, Ann Thorac Surg 66 (1998), S17-S19.

[35] E.M. Boyle, Jr., E.D. Verrier and B.D. Spiess, Endothelial cell injury in cardiovascular surgery: the procoagulant response, Ann Thorac Surg 62 (1996), 1549-1557. 
[36] M.L. Entman, L. Michael, R.D. Rossen et al., Inflammation in the course of early myocardial ischemia, FASEB J 5 (1991), 2529-2537.

[37] A. Herskowitz, S. Choi, A.A. Ansari and S. Wesselingh, Cytokine mRNA expression in postischemic/reperfused myocardium, Am J Pathol 146 (1995), 419-428.

[38] R.L. Engler, G.W. Schmid-Schonbein and R.S. Pavelec, Leukocyte capillary plugging in myocardial ischemia and reperfusion in the dog, Am J Pathol 111 (1983), 98-111.

[39] G. Ambrosio, J.T. Flaherty, C. Duilio et al., Oxygen radicals generated at reflow induce peroxidation of membrane lipids in reperfused hearts, J Clin Invest 87 (1991), 2056-2066.

[40] P. Golino, M. Ragni, P. Cirillo et al., Effects of tissue factor induced by oxygen free radicals on coronary flow during reperfusion, Nat Med 2 (1996), 35-40.

[41] P. Golino, M. Ragni, P. Cirillo et al., Recombinant human, active site-blocked factor VIIa reduces infarct size and no-reflow phenomenon in rabbits, Am J Physiol Heart Circ Physiol 278 (2000), H1507-H1516.

[42] P. Golino, P. Cirillo, P. Calabro' et al., Expression of exogenous tissue factor pathway inhibitor in vivo suppresses thrombus formation in injured rabbit carotid arteries, $\mathrm{J} \mathrm{Am} \mathrm{Coll} \mathrm{Cardiol}$ 38 (2001), 569-576.

[43] C.F. Toombs, S. McGee, W.E. Johnston and J. VintenJohansen, Myocardial protective effects of adenosine. Infarct size reduction with pretreatment and continued receptor stimulation during ischemia, Circulation 86 (1992), 986-994.

[44] J.H. Erlich, E.M. Boyle, J. Labriola et al., Inhibition of the tissue factor-thrombin pathway limits infarct size after myocardial ischemia-reperfusion injury by reducing inflammation, Am J Pathol 157 (2000), 1849-1862.

[45] A.J. Chong, T.H. Pohlman, C.R. Hampton, A. Shimamoto, N. Mackman and E.D. Verrier, Tissue factor and thrombin mediate myocardial ischemia-reperfusion injury, Ann Thorac Surg 75 (2003), S649-S655.

[46] G. Kaplanski, M. Fabrigoule, V. Boulay et al., Thrombin in- duces endothelial type II activation in vitro: IL-1 and TNFalpha-independent IL-8 secretion and E-selectin expression, $J$ Immunol 158 (1997), 5435-5441.

[47] R. Kranzhofer, S.K. Clinton, K. Ishii, S.R. Coughlin, J.W. Fenton and P. Libby, Thrombin potently stimulates cytokine production in human vascular smooth muscle cells but not in mononuclear phagocytes, Circ Res 79 (1996), 286-294.

[48] Y. Sugama, C. Tiruppathi, K. offakidevi, T.T. Andersen, J.W. Fenton and A.B. Malik, Thrombin-induced expression of endothelial P-selectin and intercellular adhesion molecule-1: a mechanism for stabilizing neutrophil adhesion, J Cell Biol 119 (1992), 935-944.

[49] X.L. Ma, P.S. Tsao and A.M. Lefer, Antibody to CD-18 exerts endothelial and cardiac protective effects in myocardial ischemia and reperfusion, J Clin Invest 88 (1991), 1237-1243.

[50] A.J. Palazzo, S.P. Jones, W.G. Girod, D.C. Anderson, D.N. Granger and D.J. Lefer, Myocardial ischemia-reperfusion injury in, Am J Physiol 275 (1998), H2300-H2307.

51] M.A. Cunningham, E. Rondeau, X. Chen, S.R. Coughlin, S.R. Holdsworth and P.G. Tipping, Protease-activated receptor 1 mediates thrombin-dependent, cell-mediated renal inflammation in crescentic glomerulonephritis, J Exp Med 191 (2000), 455-462.

[52] H. Philippou, A. Adami, S.J. Davidson, J.R. Pepper, J.F. Burman and D.A. Lane, Tissue factor is rapidly elevated in plasma collected from the pericardial cavity during cardiopulmonary bypass, Thromb Haemost 84 (2000), 124-128.

[53] S.R. Mehta, J.W. Eikelboom, H.J. Rupprecht et al., Efficacy of hirudin in reducing cardiovascular events in patients with acute coronary syndrome undergoing early percutaneous coronary intervention, Eur Heart J 23 (2002), 117-123.

[54] K.L. Neuhaus, G.P. Molhoek, U. Zeymer et al., Recombinant hirudin (lepirudin) for the improvement of thrombolysis with streptokinase in patients with acute myocardial infarction: results of the HIT-4 trial, J Am Coll Cardiol 34 (1999), 966-973. 


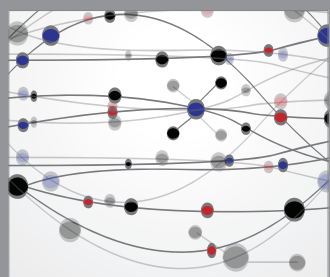

The Scientific World Journal
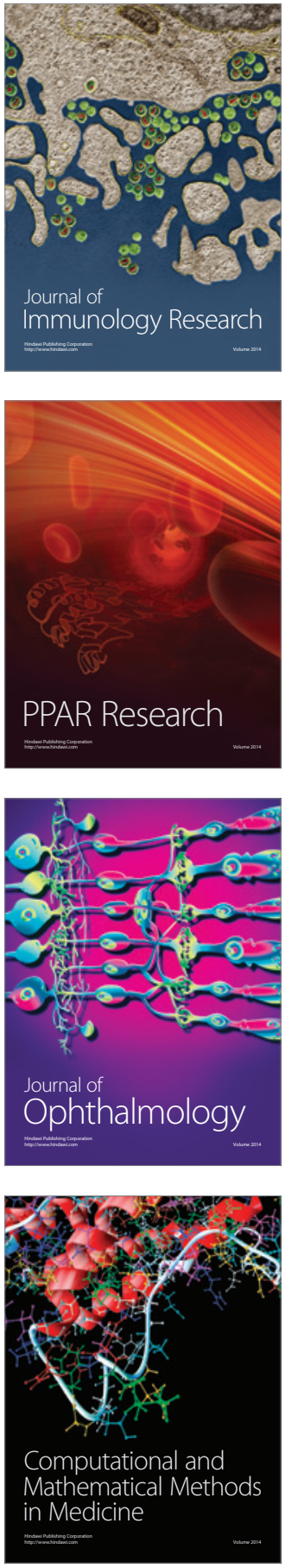

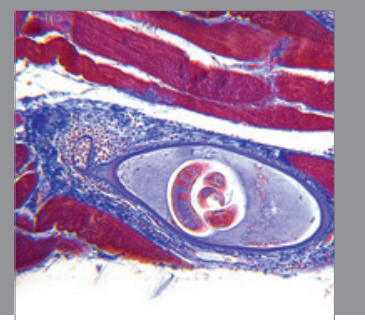

Gastroenterology

Research and Practice
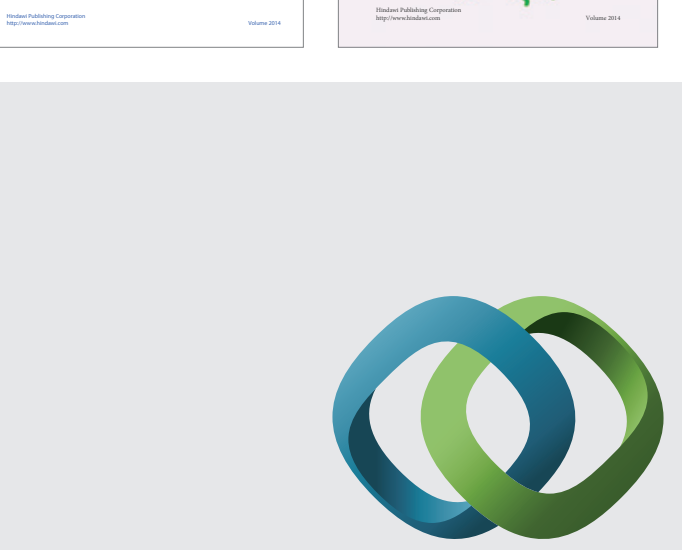

\section{Hindawi}

Submit your manuscripts at

http://www.hindawi.com
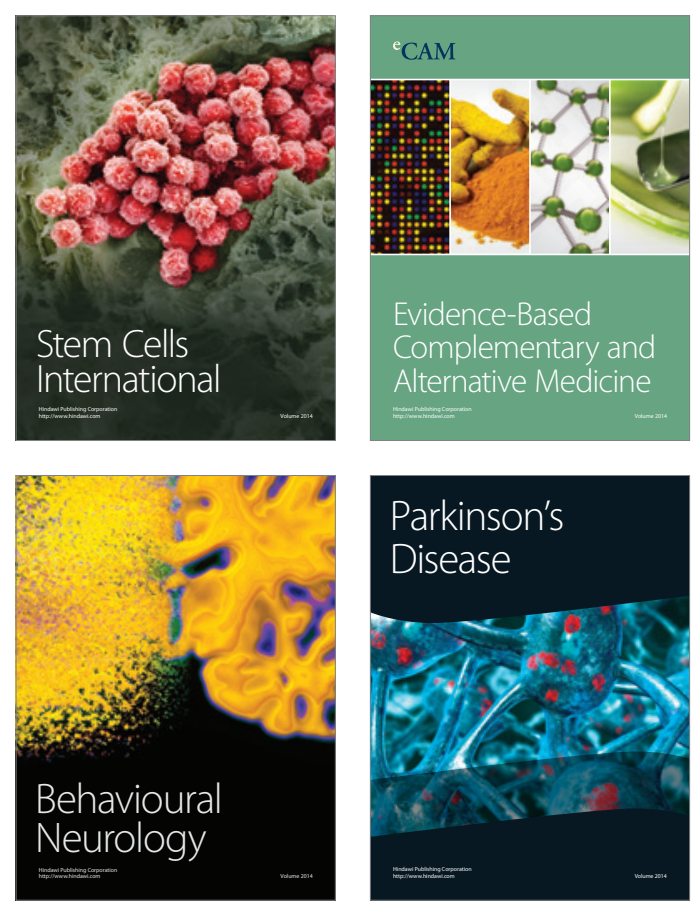

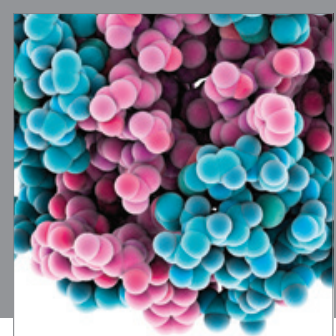

Journal of
Diabetes Research

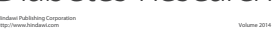

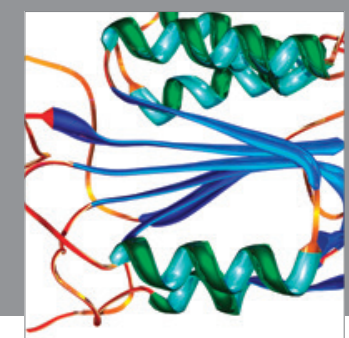

Disease Markers
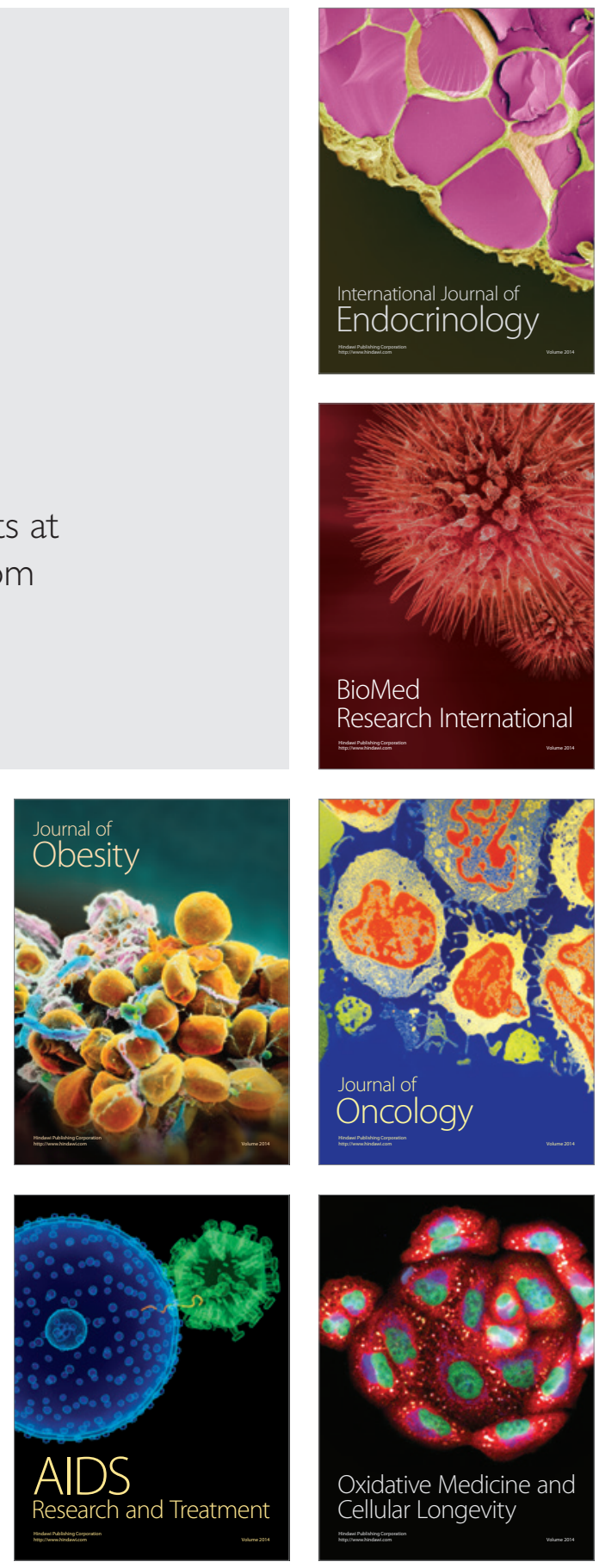